\title{
Belatacept for the prophylaxis of organ rejection in kidney transplant patients: an evidence-based review of its place in therapy
}

This article was published in the following Dove Press journal: International Journal of Nephrology and Renovascular Disease 26 May 2016

Number of times this article has been viewed

\author{
Karen L Hardinger \\ Daniel Sunderland \\ Jennifer A Wiederrich \\ Division of Pharmacy Practice and \\ Administration, School of Pharmacy, \\ University of Missouri-Kansas City, \\ Kansas City, MO, USA
}

Background: Belatacept is a novel immunosuppressive therapy designed to improve clinical outcomes associated with kidney transplant recipients while minimizing use of calcineurin inhibitors (CNIs).

Methods: We searched for clinical trials related to administration of belatacept to kidney transplant patients compared to various immunosuppression regimens, as well as for studies that utilized data from belatacept trials to validate new surrogate measures. The purpose of this review is to consolidate the published evidence of belatacept's effectiveness and safety in renal transplant recipients to better elucidate its place in clinical practice.

Results: Analysis of the results from the Belatacept Evaluation of Nephroprotection and Efficacy as First-Line Immunosuppressive Trial (BENEFIT) study, a de novo trial that compared cyclosporine (CsA)-based therapy to belatacept-based therapy in standard criteria donors, found a significant difference in mean estimated glomerular filtration rate (eGFR) of 13-15 mL/ $\mathrm{min} / 1.73 \mathrm{~m}^{2}$ and $23-27 \mathrm{~mL} / \mathrm{min} / 1.73 \mathrm{~m}^{2}$ at 1 year and 7 years, respectively. The BENEFITEXT study was similarly designed with the exception that it included extended criteria donors. Renal function improved significantly for the more intensive belatacept group in all years of the BENEFIT-EXT study; however, it was not significant in the less intensive group until 5 years after transplant. Belatacept regimens resulted in lower blood pressure, cholesterol levels, and incidence of new-onset diabetes after transplant compared to CsA-based regimens. Results from conversion of CNIs to belatacept therapy, dual therapy of belatacept with sirolimus, and belatacept with corticosteroid avoidance therapy are also included in this article.

Conclusion: The evidence reviewed in this article suggests that belatacept is an effective alternative in kidney transplant recipients. Compared to CNI-based therapy, belatacept-based therapy results in superior renal function and similar rates of allograft survival. In terms of safety, belatacept was shown to have lower incidence of hypertension, hyperlipidemia, and diabetes; however, incidence of posttransplantation lymphoproliferative disorder and the cost of belatacept may hinder use of this medication.

Keywords: costimulatory blocker, renal, BENEFIT, pharmacology, immunosuppression

\section{Introduction}

Significant advances have been made in immunosuppression therapies for kidney transplant recipients in the past few decades. Although the occurrence of early graft rejection has become rare, only small improvements have been made in terms of long-term survival. The most common cause of graft loss is chronic allograft nephropathy, and common causes of death are cardiovascular disease and cancer. However, the biggest challenge with immunosuppression therapy remains; balancing the need for immunosuppression 
to prevent graft rejection while minimizing the chance for drug toxicities, cancer, or infection.

Currently, there are five major drug classes that comprise maintenance immunosuppressive therapies. These therapies include calcineurin inhibitors (CNIs, ie, cyclosporine [CsA] and tacrolimus), mammalian target of rapamycin inhibitors (sirolimus and everolimus), antiproliferative agents (azathioprine and mycophenolic acid), costimulation blockade agents (belatacept), and corticosteroids. In accordance with the clinical practice guidelines of the Kidney Disease Improving Clinical Outcomes Work Group, compiled before the release of belatacept, CNIs are commonly used as first-line agents, in combination with mycophenolic acid with or without steroids. ${ }^{1}$ Although CNIs are effective as immunosuppressive agents, these medications are associated with nephrotoxicity, hypertension, hyperlipidemia, and new-onset diabetes after transplantation (NODAT) in kidney transplant recipients.

Belatacept is a costimulation blocker that was approved by the US Food and Drug Administration (FDA) in June 2011 for the prophylaxis of organ rejection in kidney transplant recipients. Its novel mechanism of action and the beneficial side effect profile have intrigued the transplant community. In clinical trials, belatacept administration has resulted in preserved renal function in kidney transplant recipients. Belatacept may be a better alternative to CNIs in certain patient populations.

\section{Methods}

We searched for clinical trials related to administration of belatacept to kidney transplant patients compared to various immunosuppression regimens, as well as for studies that utilized data from belatacept trials to validate new surrogate measures. The purpose of this review is to consolidate the published evidence of the effectiveness and safety of belatacept in renal transplant recipients to better understand its place in clinical practice.

\section{Pharmacology of belatacept}

Belatacept has an FDA indication for the prevention of kidney transplant rejection in combination with basiliximab induction, mycophenolate mofetil (MMF), and corticosteroids. ${ }^{2}$ It is a costimulation blocker that binds to clusters of differentiation (CD) 80 and CD86 receptors on antigen-presenting cells in order to inhibit a CD28-mediated interaction between the antigen-presenting cells and T-cells (Figure 1). Under normal circumstances, this CD28-mediated interaction results in activation of T-lymphocytes, causing an increase in cytokine production and proliferation typically associated

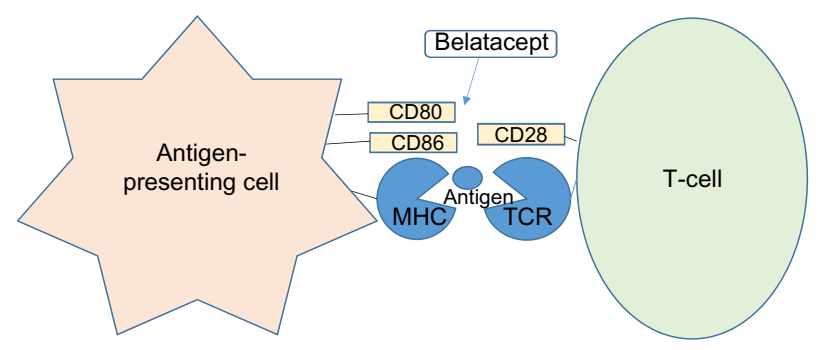

Figure I Mechanism of action of belatacept.

Notes: Belatacept binds to CD80 and CD86 receptors on antigen-presenting cells inhibiting a CD28-mediated interaction between the antigen-presenting cell and T-cells. By blocking the receptors on the antigen-presenting cell, belatacept decreases both cytokine production and proliferation of T-lymphocytes.

Abbreviations: $C D$, clusters of differentiation; MHC, major histocompatibility complex; TCR, T-cell receptor.

with immunologic rejection in kidney transplantation. Thus, by blocking the receptors on the antigen-presenting cell, belatacept decreases both cytokine production and proliferation of T-lymphocytes.

The FDA-approved dosing for belatacept, based upon safety and efficacy in Phase II and III trials, consists of a first dose of $10 \mathrm{mg} / \mathrm{kg}$ based upon actual body weight administered the day of transplantation, in addition to a second dose on day 5, or 96 hours after the first dose. ${ }^{2}$ Additional doses are given at the end of weeks $2,4,8$, and 12 . Starting at week 16 , patients should receive a $5 \mathrm{mg} / \mathrm{kg}$ dose, with subsequent doses every 4 weeks thereafter. Doses should be rounded to the nearest $12.5 \mathrm{mg}$, using the patient's actual body weight on the day of transplant, unless a change in weight of $>10 \%$ occurs during maintenance therapy. Belatacept is only available as a reconstituted solution that should be intravenously infused over 30 minutes in each session. There is no difference in the dosing of belatacept for adult and geriatric patient populations. The same approach in therapy applies to patients with hepatic or renal dysfunction. Belatacept has only been studied in adults; therefore, efficacy and safety have not been established for adolescents, children, infants, or neonates.

The use of belatacept is contraindicated in transplant patients with an unknown Epstein-Barr virus (EBV) status or those that are EBV seronegative, due to an estimated tenfold increased risk of developing posttransplant lymphoproliferative disorder (PTLD) in patients receiving belatacept who were EBV seronegative during Phase III studies. ${ }^{3}$ Therapy is also not recommended in liver transplant recipients, as it is associated with an increased risk of graft loss and death. ${ }^{2}$ Use of belatacept in higher-than-recommended or more frequent doses may increase the risk of infection, incidence of PTLD, particularly in the central nervous system, and malignancy. Caution should be used for patients with a history of active or chronic infection, as well as those with 
Table I Major adverse effects

\begin{tabular}{ll}
\hline System & Reaction (\% in clinical trials vs cyclosporine) \\
\hline Cardiovascular & Peripheral edema (34), hypertension (32), hypotension (I8) \\
Central nervous system & Fever (28), headache (2 I), insomnia (I5) \\
Endocrine and metabolic & Hypokalemia (2I), hyperkalemia (20), hypophosphatemia (I9), lipid metabolism disorder (I9), hyperglycemia (I6), \\
& hypocalcemia (I3), hypercholesterolemia (II) \\
Gastrointestinal & Diarrhea (39), constipation (33), nausea (24), vomiting (22), abdominal pain (I9) \\
Genitourinary & Urinary tract infection (37), dysuria (II) \\
Hematology and oncology & Anemia (45), leukopenia (20) \\
Infection & Increased susceptibility (72-82, serious 24-36), herpes (4-I4), cytomegalovirus (I I-I3), influenza (I I) \\
Neuromuscular and skeletal & Arthralgia (I7), back pain (I3) \\
Renal & Proteinuria (I6-33), renal graft dysfunction (25), hematuria (I6), increased serum creatinine (I5) \\
Respiratory & Cough (24), upper respiratory tract infection (I5), nasopharyngitis (I3), dyspnea (I2) \\
\hline
\end{tabular}

advanced or uncontrolled diabetes mellitus. Currently, there is no standard of care for management of infections in patients receiving belatacept, although transplantation should not be performed in patients with untreated serious infections. If a patient has a serious infection while on belatacept and is due for a belatacept dose, clinicians should weigh the risk and benefits of giving or holding immunosuppression. If a patient has a serious infection and has recently received belatacept, clinicians should consider holding or reducing other maintenance immunosuppressive medications. The terminal halflife of belatacept is $8-10$ days, but the pharmacodynamic effects may last longer.

Belatacept should not be used in pregnancy unless the potential benefit of the medication outweighs the potential risk to the fetus. ${ }^{2}$ There can be excretion of the medication into breast milk, with subsequent systemic absorption by the infant; therefore, nursing while on this medication should occur with extreme caution. The adverse effects associated with infant exposure to belatacept are not known at this time.

Major adverse effects, defined as $>10 \%$ incidence in clinical trials, are listed in Table 1, organized by organ system classification. Table 2 presents the minor adverse effects, defined as $<10 \%$ incidence, and organized in the same manner. Postmarketing surveillance has resulted in confirmed cases of aspergillosis, encephalitis, PTLD, cryptococcal meningitis, and progressive multifocal leukoencephalopathy (PML) in $<1 \%$ of patients treated with belatacept therapy.

Grinyó et al ${ }^{4}$ analyzed the 2-year safety profile of belatacept in kidney transplant recipients, using pooled data from a Phase II study of belatacept ${ }^{5}$ and from the Belatacept Evaluation of Nephroprotection and Efficacy as First-Line Immunosuppressive Trial (BENEFIT) ${ }^{6}$ and BENEFIT-EXT ${ }^{7}$ studies $(n=1,425)$. A total of 16 cases of PTLD occurred within the first 18 months after transplant. Of these 16 cases, eight occurred in more intensive (MI, Figure 2) belatacepttreated patients $(2 \%)$, six in less intensive (LI) belatacepttreated patients (1\%), and two in CsA-treated patients $(0.4 \%)$. PTLD occurrence among belatacept-treated patients was found in the renal allograft $(\mathrm{n}=5)$ and the central nervous system (CNS) ( $\mathrm{n}=9$ ). PTLD occurred more frequently in patients who received antilymphocyte therapy in the MI belatacept therapy group (MI: 5.7\%, LI: $0 \%$, CsA: $0 \%$ ) and in

Table 2 Minor adverse effects

\begin{tabular}{|c|c|}
\hline System & Reaction (\% in clinical trials vs cyclosporine) \\
\hline Cardiovascular & Thrombosis of arteriovenous graft $(<10)$, atrial fibrillation $(<10)$ \\
\hline Central nervous system & Anxiety (10), Guillain-Barre Syndrome $(<10)$, dizziness $(9)$ \\
\hline Dermatologic & Alopecia $(<10)$, hyperhidrosis $(<10)$, acne vulgaris $(8)$ \\
\hline Endocrine and metabolic & New-onset diabetes (5-8), hypomagnesemia (7), hyperuricemia (5) \\
\hline Gastrointestinal & Stomachitis $(<10)$, upper abdominal pain $(9)$ \\
\hline Genitourinary & Urinary incontinence $(<10)$ \\
\hline Hematology/oncology & Hematoma $(<10)$, lymphocele $(<10)$, neutropenia $(<10)$, malignant neoplasm $(4)$, nonmelanoma $(2)$ \\
\hline Immunologic & Antibody development (2) \\
\hline Infection & Polyoma virus $(3-4)$ \\
\hline Neuromuscular and skeletal & Musculoskeletal pain $(<10)$, tremor $(8)$ \\
\hline Renal & $\begin{array}{l}\text { Acute renal failure }(<10) \text {, chronic allograft nephropathy }(<10) \text {, hydronephrosis, renal insufficiency, renal artery } \\
\text { stenosis, renal tubular necrosis }(9), \text { BK-associated renal disease }(I)\end{array}$ \\
\hline Respiratory & Bronchitis (I0), tuberculosis $(I-2)$ \\
\hline Miscellaneous & Infusion site reaction (5) \\
\hline
\end{tabular}




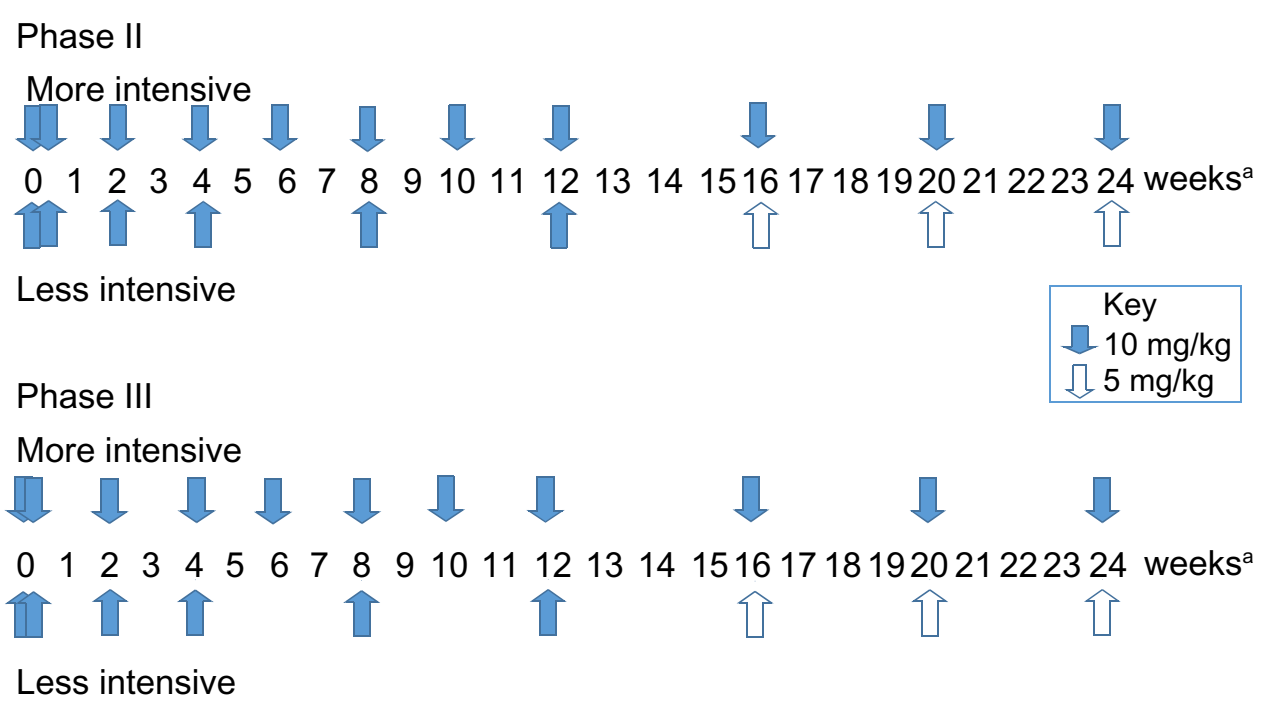

Figure 2 Belatacept dosing regimens in clinical studies by week.

Notes: Phase II more intensive: $10 \mathrm{mg} / \mathrm{kg}$ on days I, 5, and I5; then every 2 weeks through Month 3, and then every 4 weeks through Month 6; thereafter, $5 \mathrm{mg} / \mathrm{kg}$ every 4 weeks or 8 weeks. Phase II less intensive: $10 \mathrm{mg} / \mathrm{kg}$ on days I, 5, I5, 29, 57, and 85; then, $5 \mathrm{mg} / \mathrm{kg}$ every 4 weeks or 8 weeks thereafter. Phase III more intensive: $10 \mathrm{mg} / \mathrm{kg}$ on days I and 5; then every 2 weeks through Month 3; and then every 4 weeks through Month 6; thereafter, $5 \mathrm{mg} / \mathrm{kg}$ every 4 weeks. Phase III less intensive: $10 \mathrm{mg} / \mathrm{kg}$ on days $\mathrm{I}$ and 5; then at the end of weeks $2,4,8$, and I2; next, $5 \mathrm{mg} / \mathrm{kg}$ starting on week I6, then every 4 weeks thereafter. ${ }^{\mathrm{a}}$ Then, $5 \mathrm{mg} / \mathrm{kg}$ every 4 weeks.

patients who were EBV seronegative at baseline. The overall frequency of infections was found to be similar between all treatment groups $(\sim 80 \%)$. Polyomavirus, fungal infections, and CNS infections occurred less frequently in the LI belatacept treatment group. Overall, belatacept was well tolerated with few adverse side effects, with the LI belatacept regimen offering a better safety profile.

Belatacept displays linear kinetics in which the drug reaches steady state 8 weeks posttransplant and 6 months with maintenance therapy. ${ }^{2}$ Peak concentrations of $247 \pm 68 \mu \mathrm{g} / \mathrm{mL}$ and $139 \pm 28 \mu \mathrm{g} / \mathrm{mL}$ occur after administration of multiple $10 \mathrm{mg} / \mathrm{kg}$ and $5 \mathrm{mg} / \mathrm{kg}$ doses, respectively. Accumulation percentage doubles with once-monthly infusion of the highdose regimen $(20 \%)$ compared to late-phase maintenance therapy $(10 \%)$. Volume of distribution for transplant patients at steady state is $0.11 \mathrm{~L} / \mathrm{kg}$. The average total body clearance is $0.49 \pm 0.13 \mathrm{~mL} / \mathrm{kg} / \mathrm{h}$ and increases in direct relation to increased body weight. Hepatic and renal function did not influence clearance of the drug. The elimination half-life is $9.8 \pm 3.2$ days and $8.2 \pm 2.4$ days for $10 \mathrm{mg} / \mathrm{kg}$ and $5 \mathrm{mg} / \mathrm{kg}$ doses, respectively.

The use of live vaccines (ie, measles, mumps, and rubella [MMR], intranasal influenza, varicella, yellow fever, and TY21a typhoid fever vaccines) should be avoided during treatment with all immunosuppressants, including belatacept, due to an increased risk of infection. ${ }^{2}$ Echinacea may decrease the overall immunosuppressive effect of belatacept, while the combination of belatacept and other immunosuppressive medications can increase risk of infection, due to greater immunosuppression.

Due to an increased risk of PTLD, along with the potential of progressive PML reported in Phase III belatacept clinical trials, a risk evaluation and mitigation strategy (REMS) program was developed in collaboration with the FDA. ${ }^{3}$ Belatacept's REMS program informs health care professionals and patients of the increased risk of PTLD and PML. The program also provides a medication guide to patients receiving treatment upon hospital discharge and with every subsequent infusion. Prior to each infusion, a checklist assessing the patient's clinical status must be completed to determine whether continuation with belatacept therapy based on neurological, cognitive, and behavioral changes would be appropriate. The manufacturer, Bristol-Myers Squibb (NY, USA), developed the Evaluating Nulojix ${ }^{\circledR}$ Long-Term Safety in Transplant (ENLiST) registry to further determine the incidence of PTLD, PTLD affecting the CNS, and PML in patients who are EBV seropositive. ${ }^{8}$ Registration of patients into the REMS program is required by the FDA; however, enrollment into the ENLiST registry is optional.

\section{Clinical trials Phase II de novo trial}

Vincenti et $\mathrm{al}^{5}$ conducted a prospective, randomized, parallel-group, partially blinded, multicenter, active control, noninferiority Phase II trial to determine whether belatacept demonstrated no significant difference in terms of the 
incidence of acute rejection at 6 months compared to CsA in kidney transplant patients. The study included patients who received a kidney transplant from non-human leukocyte antigen (HLA)-identical living donor or deceased donor but excluded patients with previous treatment with basiliximab, those with cold ischemia time $>36$ hours, donors older than 60 years of age, or those who received organ donation from a patient after cardiac death. High-risk patients, including those with retransplants, and patients with a panel reactive antibody score $>20 \%$ could make up no more than $10 \%$ of the study population. At the end of enrollment, only one patient was retransplanted and five patients had elevated panel reactive antibodies $(2.8 \%)$. Furthermore, 218 patients were randomized in a 1:1:1 fashion among three treatment arms: MI belatacept regimen $(n=74)$, LI belatacept regimen $(n=71)$, or CsA (CsA, $n=73$ ) for primary immunosuppression therapy. Dosing of belatacept occurred in an early and a late phase for the two groups (Figure 2). Early-phase dosing was $10 \mathrm{mg} / \mathrm{kg}$ intravenously compared to $5 \mathrm{mg} / \mathrm{kg}$ in the late phase. The MI group received early-phase dosing for 6 months and more frequent dosing, while the LI group received early-phase dosing for 3 months. CsA doses fluctuated to maintain specific goal trough concentrations, $150-400 \mathrm{ng} / \mathrm{mL}$. Monitoring trough goals and adjusting dosing regimens were not blinded in the study. All patients received induction therapy with basiliximab, high-dose steroids that were tapered to $10 \mathrm{mg} / \mathrm{d}$ by day 101, along with maintenance therapy of MMF $2 \mathrm{~g}$ daily.

The rate of acute rejection did not differ statistically among the treatment regimens, with five, four, and six acute rejection events occurring in the MI, LI, and CsA groups, respectively. The measured glomerular filtration rates (GFRs) at 12 months were $66.3 \mathrm{~mL} / \mathrm{min} / 1.73 \mathrm{~m}^{2}$, $62.1 \mathrm{~mL} / \mathrm{min} / 1.73 \mathrm{~m}^{2}$, and $53.5 \mathrm{~mL} / \mathrm{min} / 1.73 \mathrm{~m}^{2}$ in the $\mathrm{MI}$, LI, and CsA groups, respectively. There was a statistically significant difference in the GFR for MI compared to that for CsA $(P=0.01)$ and GFR for LI compared to that for CsA $(P=0.04)$. Belatacept groups also showed a difference in the incidence of 12-month protocol-biopsy-proven chronic allograft nephropathy versus the incidence with the CsA regimen $(\mathrm{MI}=29 \%, \mathrm{LI}=20 \%$, and $\mathrm{CsA}=44 \%$ ), while graft and patient survival remained similar among the regimens. Frequency of infection remained similar between the study groups; however, malignancy did develop in two patients within the MI group and two in the CsA group. There were three cases of PTLD confirmed during the study; two of the patients who were diagnosed received MI belatacept therapy but had switched to tacrolimus at the time of diagnosis. Similar lipid and blood pressure profiles were seen in the treatment groups at 12 months. Limitations of this trial included the use of less contemporary immunosuppressive regimens, including $\mathrm{CsA}$, at relatively high trough concentrations (150-300 ng/mL beyond 2 months). Results of this study suggest that belatacept has similar efficacy for preventing acute rejection in patients who have received a kidney transplant as with traditional CsA maintenance therapy in combination with other immunosuppressive agents while maintaining a higher measured GFR 12 months after transplant. Five-year follow-up of this study revealed improvement in renal function of the belatacept arm (GFR, $74 \mathrm{~mL} / \mathrm{min} \mathrm{MI}$ and $76 \mathrm{~mL} / \mathrm{min} \mathrm{LI}$ ) versus stable renal function in the CsA arm $(53 \mathrm{~mL} / \mathrm{min}) .{ }^{9}$ Additionally, no new cases of PTLD occurred.

\section{Phase III BENEFIT studies}

The BENEFIT 6 study was a Phase III, randomized, activecontrolled, parallel-group study to determine the efficacy of a belatacept-based regimen compared to CsA-based therapy 12 months after transplant. Only living and standard criteria donor (SCD) recipients were included in this study. This was a three-treatment-arm study in which 666 patients were randomized 1:1:1 to either MI belatacept regimen (Figure 2, $\mathrm{n}=225)$, LI belatacept regimen $(\mathrm{n}=230)$, or a CsA regimen $(n=231)$. Patients and investigators were aware of the medication assignment for each patient but were blinded to the dose of belatacept. The primary outcome assessed patient and graft survival, acute rejection, and renal impairment. The renal impairment composite end point was determined if the estimated glomerular filtration rate (eGFR) was $<60 \mathrm{~mL} / \mathrm{min} / 1.73 \mathrm{~m}^{2}$ at $12 \mathrm{months}$ or if there was a decline of $>10 \mathrm{~mL} / \mathrm{min} / 1.73 \mathrm{~m}^{2}$ in the eGFR between the third month and the 12 th month. Figure 3 details the average eGFR for the original BENEFIT study, as well as for the follow-up years $2,3,5$, and 7 . Renal function was superior in both belatacept treatment arms compared to CsA at 12 months $(P<0.001)$ with belatacept, resulting in an eGFR value that was higher by $13-15 \mathrm{~mL} / \mathrm{min} / 1.73 \mathrm{~m}^{2}$. Preserved renal function maintained statistical difference for the belatacept groups in all subsequent long-term efficacy studies.

Patient and graft survival were not statistically different between treatment groups at 12 months. The percentage of acute rejection rates was higher for patients treated with belatacept, which reached statistical difference for the MI and CsA regimens (MI: 22\%; CsA: 7\%) but not between the LI and CsA regimens (LI: 17\%; CsA: 7\%). Patients on the $\mathrm{MI}$ regimen also experienced more Banff grade IIB rejection in comparison to patients on CsA. However, among those 


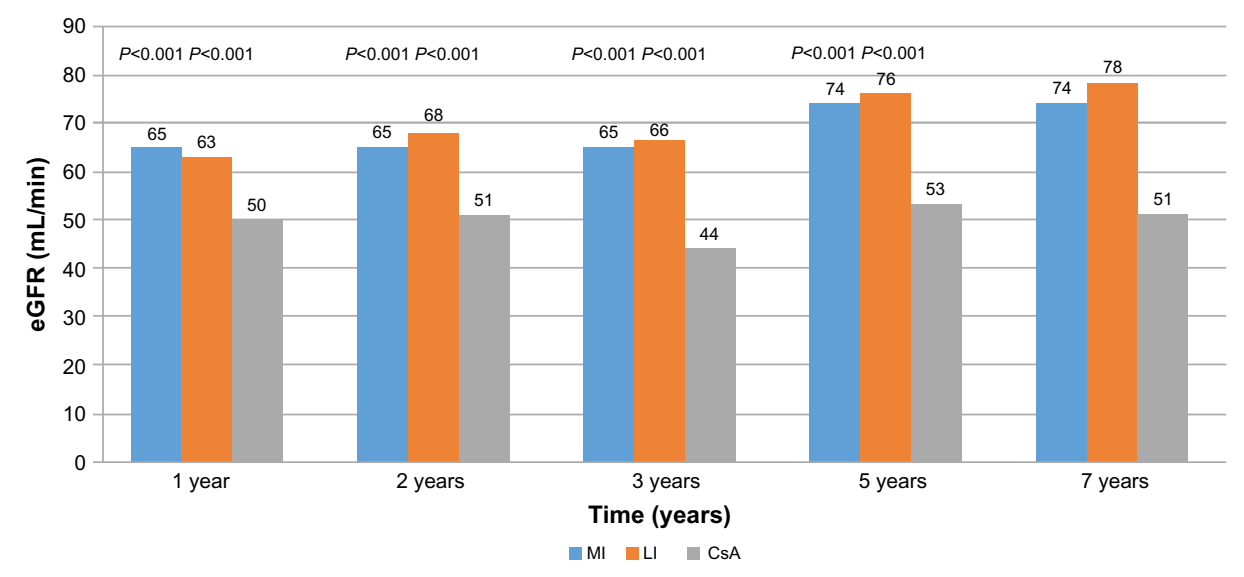

Figure 3 Mean eGFR in the BENEFIT study.

Notes: Reported $P$-values were generated for each belatacept group in comparison to the cyclosporine control group. If values for renal function did not have corresponding $P$-values, they were either absent in the study or not reported. Data from Vincenti et al, ${ }^{6,13}$ Larsen et al, ${ }^{10}$ Pestana et al, ${ }^{14}$ and Charpentier et al. ${ }^{15}$

Abbreviations: BENEFIT, Belatacept Evaluation of Nephroprotection and Efficacy as First-Line Immunosuppressive Trial; MI, more intensive; LI, less intensive; CsA, cyclosporine; eGFR, estimated glomerular filtration rate.

patients with acute rejection, a similar number of patients in each group survived with a functioning graft (45/48 MI; 36/39 LI; 15/16 CsA). Systolic and diastolic blood pressures were significantly lower for patients treated with belatacept, in addition to the use of lower number of antihypertensive agents for control, compared to the CsA group of patients. Belatacept treatment groups had a statistically significant change in the cholesterol panel (non-high-density lipoprotein, triglycerides) from baseline; however, NODAT rates were similar among the three groups at 12 months. Incidence of side effects occurred similarly within the groups, along with the frequency of bacterial, viral, and fungal infections.

Long-term safety and efficacy have been assessed for the BENEFIT study, with study analysis completed at 2 years, 3 years, 5 years, and 7 years after transplantation. ${ }^{9-13}$ Superiority of the renal function with belatacept regimens has been sustained in all follow-up BENEFIT studies, with the LI regimen favored to provide a balance between safety and efficacy. A mean difference of $14-18 \mathrm{~mL} / \mathrm{min} / 1.73 \mathrm{~m}^{2}$, $11-12 \mathrm{~mL} / \mathrm{min} / 1.73 \mathrm{~m}^{2}, 21-23 \mathrm{~mL} / \mathrm{min} / 1.73 \mathrm{~m}^{2}$, and 24-25 mL/min/1.73 $\mathrm{m}^{2}$, respectively, was seen between belatacept regimens and CsA at 24 months, 36 months, 60 months, and 84 months posttransplant. Allograft survival continued to be similar for the belatacept and CsA regimens despite belatacept regimens having an increased incidence of acute rejection early on in the study. A better cardiovascular and metabolic profile was seen in the belatacept regimens compared to CsA in terms of hypertension, lipid panel (total cholesterol, low-density lipoprotein, and triglycerides), and NODAT. The incidence of adverse events along with fungal infections and development of malignancies remained comparable among the groups according to the 7-year follow-up results.

The final results of the BENEFIT study were published in 2016. ${ }^{13}$ Of the 660 patients initially treated, 153 of 219 (in MI group), 163 of 226 (in LI group), and 131 of 215 (in CsA group) were followed for 84 months. Of these patients, only 128 (MI group), 136 (LI group), and 92 (CsA group) completed the 84-month study. Many patients were ineligible to continue, declined the extension study, withdrew consent, had an adverse effect, died, were lost to follow-up, or were nonadherent and not included in the study. Of the patients who could be evaluated at 84 months, the risk of death or graft loss was $43 \%$ lower among patients treated with belatacept compared with those on CsA. Kaplan-Meier estimates for rates of graft loss among patients receiving MI belatacept, those receiving LI belatacept, and those receiving CsA, with censoring of data for death, were $4.7 \%$, $4.1 \%$, and $4.6 \%$, respectively, at month $36 ; 4.7 \%, 4.1 \%$, and $9.8 \%$, respectively, at month 60 ; and $4.7 \%, 5.4 \%$, and $9.8 \%$, respectively, at month 84 . At month 60 , the hazard ratio for the comparison of allograft losses between MI belatacept and CsA was 0.56 (95\% confidence interval [CI]: 0.26-1.23; $P=0.12$ ), and the hazard ratio for the comparison of the same between LI belatacept and CsA was 0.49 (95\% CI: 0.22-1.09; $P=0.07)$. The incidence of development of donor-specific antibodies was $1.9 \%$ in the MI regimen, $4.6 \%$ in the LI regimen, and $17.8 \%$ in the CsA arm $(P<0.001)$.

\section{Phase III BENEFIT-EXT studies}

The BENEFIT-EXT study is one of the largest trials evaluating the efficacy of belatacept compared to CsA as first-line 
immunosuppression for patients with extended criteria donor (ECD) kidneys, and it is an extension of the original BENEFIT study. ${ }^{7}$ The primary objective of the study was to assess composite patient and graft survival and composite renal impairment between a belatacept-based regimen and a CsA-based regimen. Other immunosuppression and trial designs were similar to the BENEFIT study. Patients were randomized 1:1:1 to MI belatacept regimen (Figure 2, $\mathrm{n}=184)$, LI belatacept regimen $(\mathrm{n}=175)$, or a CsA regimen $(\mathrm{n}=184)$.

One-year patient and graft survival results were noninferior to CsA in both MI and LI belatacept arms (86\% MI, $89 \% \mathrm{LI}$, and $85 \% \mathrm{CsA})$. The incidence of acute rejection at 1 year was similar in each group (18\% MI, 18\% LI, and 14\% CsA). Results of this study at 1 year, 3 years, ${ }^{14} 5$ years, ${ }^{15}$ and 7 years ${ }^{16}$ show that belatacept may be more beneficial in preserving renal function. ${ }^{13-15}$ Figure 4 details the results of each study in terms of renal function (eGFR), organized by number of years posttransplant. At the end of 1 year, the eGFR of the MI belatacept group was significantly higher than that of the CsA group: $50 \pm 25 \mathrm{~mL} / \mathrm{min}$ MI versus $45 \pm 21 \mathrm{~mL} / \mathrm{min}$ CsA; $P=0.0083$. The eGFR of the LI belatacept group was not significantly higher than that of the CsA group: $52 \pm 22 \mathrm{~mL} /$ $\min \mathrm{LI}$ versus $45 \pm 21 \mathrm{~mL} / \mathrm{min} \mathrm{CsA} ; P=0.104$. A similar trend was observed at 2 years (MI vs CsA: $P=0.028$; and LI vs CsA: $P=0.180$ ). Five-year data showed a statistically significant improvement in the eGFR of both the MI and LI groups when compared to the CsA group (MI: $56 \mathrm{~mL} / \mathrm{min}, P=0.0108$; LI: $59 \mathrm{~mL} / \mathrm{min}, P<0.001$; CsA: $45 \mathrm{~mL} / \mathrm{min}$ ). Seven-year data demonstrated a similar trend in renal function, although significance was not mentioned. ${ }^{16}$ In summary, at the end of
Year 1, the mean eGFR in patients receiving belatacept was 6-8 $\mathrm{mL} / \mathrm{min}$ higher than that in the CsA group. This value progressed to an average difference of $11 \mathrm{~mL} / \mathrm{min}$ by Year 3 and $11-14 \mathrm{~mL} / \mathrm{min}$ by Year 5 .

Other trends in therapy with belatacept were also observed in these studies. For all studies, the incidence of adverse drug reactions was similar between both belatacept groups and the CsA group. Patients being treated with belatacept were also seen to have lower systolic and diastolic blood pressures. The 3 -year results of the study conducted by Pestana et $\mathrm{al}^{14}$ indicated that patients taking belatacept used fewer medications for hypertension and hyperlipidemia. The authors also commented that NODAT incidence was less in the MI belatacept treatment group and that CsA patients had a higher incidence of donorspecific antibodies. Overall, all studies under the BENEFITEXT series agreed that belatacept is associated with improved renal function after transplant compared with CsA.

A post hoc analysis of patients with preexisting diabetes in the BENEFIT and BENEFIT-EXT studies was conducted to access outcomes in this high-risk population $(n=336) .{ }^{17}$ Patients were considered to have diabetes before transplantation if they reported a medical history of diabetes or were taking insulin or oral antidiabetic medication at the time of transplantation. At 1 year, patients surviving with a functioning graft (90.4\% MI, 92.8\% LI, and $80.8 \% \mathrm{CsA})$ and measured GFR (MI: $59.8 \mathrm{~mL} / \mathrm{min} / 1.73 \mathrm{~m}^{2}$; LI: $62.5 \mathrm{~mL} /$ $\mathrm{min} / 1.73 \mathrm{~m}^{2}$; CsA: $45.4 \mathrm{~mL} / \mathrm{min} / 1.73 \mathrm{~m}^{2}$ ) were similar. Higher rates of acute rejection were observed with belatacept (22.8\% MI, 20.6\% LI, and $14.4 \% \mathrm{CsA}$ ), but cardiovascular risk profile was better for patients on belatacept than for those on CsA.

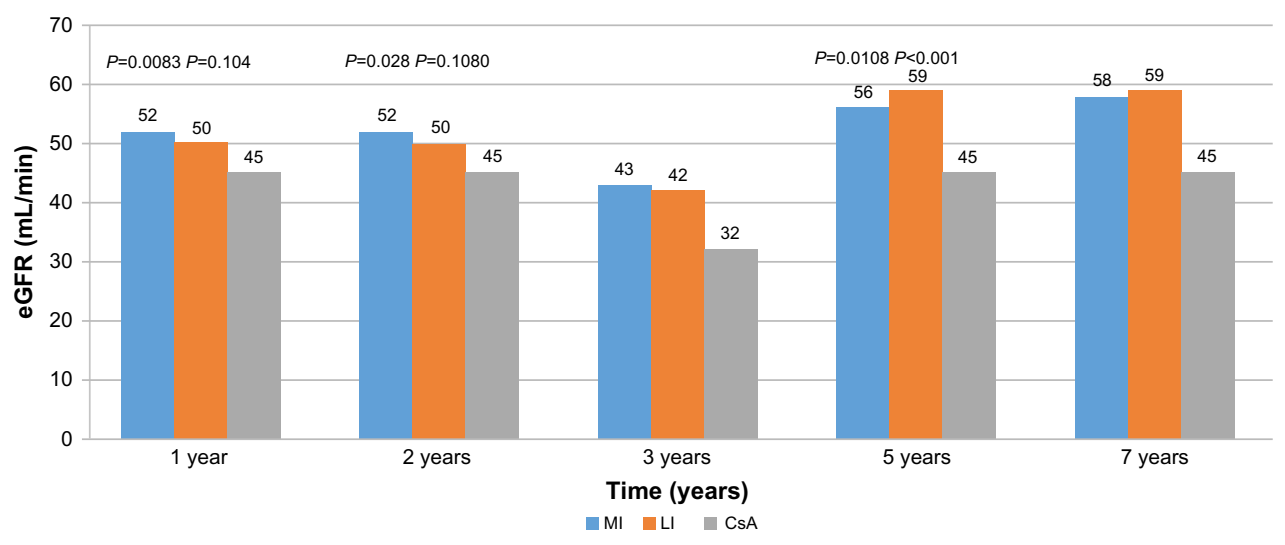

Figure 4 Mean eGFR in the BENEFIT-EXT study.

Notes: Reported $P$-values were generated for each belatacept group in comparison to the cyclosporine control group. If values for renal function did not have corresponding P-values, they were either absent in the study or not reported. Data from Durrbach et al, ${ }^{7}$ Larsen et al, ${ }^{10}$ Rostaing et al, ${ }^{12}$ Vincenti et al, ${ }^{13}$ and Pestana et al. ${ }^{14}$

Abbreviations: BENEFIT-EXT, Belatacept Evaluation of Nephroprotection and Efficacy as First-Line Immunosuppressive Trial, extended criteria; MI, more intensive; LI, less intensive; CsA, cyclosporine; eGFR, estimated glomerular filtration rate. 


\section{$\mathrm{CNI}$ and corticosteroid avoidance trial}

One limitation of the BENEFIT and BENEFIT-EXT studies is that the trials did not have external validity, as most transplant centers use rabbit anti-thymocyte globulin, tacrolimus, or mycophenolic acid with or without corticosteroids. Thus, a subsequent trial designed by Ferguson et $\mathrm{al}^{18}$ attempted to study a more current steroid-sparing strategy. The 1-year, randomized, active-controlled, open-label, Phase II exploratory study tested the efficacy and safety of dual maintenance immunosuppressive therapy while avoiding the use of maintenance corticosteroids. Eighty-nine patients who received a non-HLA-identical living or deceased SCD organ were randomized 1:1:1 to receive a maintenance regimen of belatacept plus MMF, belatacept plus sirolimus, or tacrolimus plus MMF. Rabbit anti-thymocyte globulin, at a total dose of $6 \mathrm{mg} / \mathrm{kg}$, was administered as induction therapy along with a 4-day methylprednisolone taper. Belatacept dosing was based upon the Phase III MI regimen due to the lack of steroids: $10 \mathrm{mg} / \mathrm{kg}$ intravenously on day 1 and day 5 , then every other week for 12 weeks, and then every 4 weeks for 12 weeks; and finally, $5 \mathrm{mg} / \mathrm{kg}$ every 4 weeks thereafter. Dosing for sirolimus and tacrolimus started at $5 \mathrm{mg} / \mathrm{kg} / \mathrm{d}$ and $0.1 \mathrm{mg} / \mathrm{kg} / \mathrm{d}$, respectively, and was adjusted to achieve prespecified therapeutic concentrations. MMF was initiated at $1 \mathrm{~g}$ twice daily, with the option to increase frequency if not tolerated or to switch to mycophenolic acid. During the study, discontinuation or switching of regimens occurred in eight (24\%), 12 (46\%), and two (7\%) of the belataceptMMF, belatacept-sirolimus, and tacrolimus-MMF patients, respectively.

The primary outcome showed no statistically significant difference in the number of acute rejections at 6 months among belatacept-MMF, belatacept-sirolimus, and tacrolimus-MMF (four out of 33 events, 12\%; one out of 26 events, $4 \%$; and one out of 30 events, $3 \%$, respectively). Of note, three patients switched from the belatacept-MMF group to tacrolimusMMF. Patient and graft survival rates were similar among the groups, with $91 \%, 92 \%$, and $100 \%$ of patients surviving with graft function in place at 12 months. There was a statistically significant difference for the belatacept groups, which showed a higher 12-month mean calculated eGFR of $8-10 \mathrm{~mL} / \mathrm{min} / 1.73 \mathrm{~m}^{2}$ versus the tacrolimus arm. Patients treated in the tacrolimus group experienced a higher percentage of steroid-free days compared to belatacept-MMF and belatacept-sirolimus groups $(93 \%, 73 \%$, and $77 \%$, respectively). The cardiovascular and metabolic profiles did not demonstrate clinically or statistically significant differences among the three groups. Tacrolimus was associated with a higher incidence of tremor (seven out of $30,22 \%$ ), compared to belatacept-MMF (one out of 33, 3\%); however, aphthous stomatitis and proteinuria were reported in the belatacept groups but not in the tacrolimus groups. Limitations of the trial include its short time frame, small size, lack of statistical power, and high drug conversion or discontinuation rates. The authors concluded that the use of a dual immunosuppressive regimen that avoided corticosteroids and CNIs resulted in an acceptable rate of acute rejection at 6 months and an improved renal profile, while maintaining a similar safety profile among the three regimens. Additionally, this trial demonstrated that belatacept can be safely used in patients treated with rabbit anti-thymocyte globulin.

\section{Meta-analysis}

In a recent meta-analysis, four studies ${ }^{5-7,18}$ that investigated 1,516 patients were included. ${ }^{19}$ For up to 3 years following transplant, belatacept- and CNI-treated recipients were at similar risks of dying, graft loss and returning to dialysis, as well as having acute rejection, malignancy, or PTLD (Table 3). Belatacept-treated patients were less likely to have chronic kidney scarring and had improved measured GFR when compared to CNI-treated recipients. Blood pressure was lower, lipid profile was better, and incidence of NODAT was reduced by $39 \%$ among belatacept-treated versus CNI-treated recipients.

\section{Steroid-sparing, dual maintenance therapy trial}

One study has shown promise for the use of belatacept as monotherapy. In a study conducted by Kirk et al, ${ }^{20}$ the safety and efficacy of a therapy regimen using donor bone marrow infusions $(n=9)$, alemtuzumab induction, monthly belatacept,

Table 3 Meta-analysis results

\begin{tabular}{ll}
\hline Factor & Relative effect $^{\text {a }}(\mathbf{9 5 \%} \mathbf{~ C l})$ \\
\hline Death & $0.75(0.39-1.44)$ \\
Loss of kidney transplant & $0.91(0.61-1.38)$ \\
Acute rejection & $1.56(0.85-2.86)$ \\
Chronic kidney scarring & $0.72(0.55-0.94)$ \\
Malignancy & $1.00(0.58-1.72)$ \\
PTLD & $2.79(0.61-12.66)$ \\
New-onset diabetes & $0.61(0.40-0.93)$ \\
Delayed graft function & $0.93(0.79-1.09)$ \\
Presence of donor-specific antibodies & $0.14(0.01-1.31)$ \\
\hline
\end{tabular}

Notes: ${ }^{\text {TT }}$ The relative effect of death is 0.75 . Therefore, belatacept-treated patients are 0.75 times less likely to suffer death than cyclosporine-treated patients, based on the meta-analysis. Adapted with permission from Masson P, Henderson L, Chapman JR, Craig JC, Webster AC. Belatacept for kidney transplant recipients. Cochrane Database Syst Rev. 2014. Copyright $\odot 2014$ The Cochrane Collaboration. Published by John Wiley \& Sons, Ltd. ${ }^{19}$

Abbreviations: $\mathrm{Cl}$, confidence interval; PTLD, posttransplant lymphoproliferative disorder. 
and daily sirolimus were evaluated in 20 successful kidney de novo transplant recipients. Only EBV-positive, first, non-HLA-identical, live donor allografts were included in the trial. A dose of $10 \mathrm{mg} / \mathrm{kg}$ of belatacept was given on the first postoperative day, repeated on days 3,7 , and 14, then every 2 weeks for four additional doses, and then monthly through month 6 . Thereafter, belatacept was given at a dose of $5 \mathrm{mg} / \mathrm{kg}$ monthly. Seventeen patients tolerated sirolimus therapy. The other three were switched to mycophenolic acid due to adverse effects caused by sirolimus.

The results of this study showed that mean serum creatinine was $1.1 \pm 0.1 \mathrm{mg} / \mathrm{dL}$ (eGFR $89 \pm 4 \mathrm{~mL} / \mathrm{min}$ ) at 12 months and $1.1 \pm 0.1 \mathrm{mg} / \mathrm{dL}$ (eGFR $88 \pm 3 \mathrm{~mL} / \mathrm{min}$ ) at 36 months. In the first year of study, no patients experienced biopsy-proven acute rejection, developed donor-specific antibodies, or were readmitted for opportunistic infection or malignancy. Ten patients experienced transient, subclinical BK viremia, which resolved with reduction of sirolimus. At 1 year after transplantation, sirolimus weaning was attempted. Five of 20 patients reached successful belatacept monotherapy. It was concluded in the study that a belatacept-based regimen avoiding the use of maintenance steroids and CNIs can effectively be used. Limitations of this trial included the uncontrolled, single-center design and the presence of relatively low immunologic risk recipients.

\section{Conversion trials}

Belatacept may preserve renal function in patients who need to be converted to new therapy. A 1-year trial conducted by Rostaing et $\mathrm{al}^{21}$ and a 2-year follow-up conducted by Grinyó et $\mathrm{al}^{22}$ focused on the effects of belatacept on renal function in kidney transplant patients at 6-36 months posttransplant. In this Phase II, open-label trial, patients were randomized in a 1:1 ratio to either continuation of maintenance dose immunosuppression with CNI $(n=89)$ or switchover to a belatacept-based regimen ( $n=84)$. Other immunosuppressive therapy was maintained unless adjustments were deemed medically necessary. For the belatacept treatment group, a dose of $5 \mathrm{mg} / \mathrm{kg}$ of belatacept was given on days 1, 15, 29, 43 , and 57 , then every 4 weeks thereafter. Six patients $(7 \%)$ in the belatacept group suffered acute rejection during the first 6 months after transplant without allograft loss. At Year 1, the mean change in baseline eGFR, the primary end point, was $+7.1 \mathrm{~mL} / \mathrm{min}$ for the belatacept group and $+2.8 \mathrm{~mL} / \mathrm{min}$ for the CNI group. At 2 years, the mean change in baseline eGFR was $+8.8 \mathrm{~mL} / \mathrm{min}$ for the belatacept group and $+0.3 \mathrm{~mL} / \mathrm{min}$ for the CNI group. The overall safety profiles were similar. Limitations of this trial include the small sample size and lack of statistical power. The authors concluded that belatacept may help better preserve renal function in kidney transplant patients converted from CsA.

A small, single-arm study ${ }^{23}$ has shown promise in converting high-immunologic-risk patients (patents who were sensitized, African American, retransplanted, and with delayed graft function) to belatacept for presumed acute CNI toxicity and/or interstitial fibrosis/tubular atrophy. Six patients were switched from tacrolimus to belatacept at a median of 4 months posttransplantation. Tacrolimus was weaned, MMF was maximized, prednisone was continued, and belatacept was given at a dose of $5 \mathrm{mg} / \mathrm{kg}$ on days 1, 15, 29, 43, and 57 , then every 4 weeks. Significant improvements in renal function were noted (eGFR $23.8 \pm 12.9 \mathrm{~mL} / \mathrm{min} / 1.73 \mathrm{~m}^{2}$ before conversion to $42 \pm 12.5 \mathrm{~mL} / \mathrm{min} / 1.73 \mathrm{~m}^{2}$ at $16.5 \mathrm{months}$ postconversion; $P=0.03$ ) without new acute rejection, PTLD, or development of donor-specific antibodies.

\section{Quality measures Quality of life}

Change in a transplant patients' health-related quality of life (HRQoL) plays a significant role in determining the effects of transplantation and its implication to each patient. Dobbels et $\mathrm{al}^{24}$ conducted a study to determine the relationship between the severity of chronic kidney disease, number of reported side effects after immunosuppressive therapy, and effect of transplantation on a patients' HRQoL as it pertains to the BENEFIT and BENEFIT-EXT studies. In these studies, patients were required to complete the Medical Outcomes Study 36-Item Short Form Health Survey (SF-36) at baseline (but not before transplant) and at 12 months, 24 months, and 36 months posttransplantation. Responses to the Modified Transplant Symptom Occurrence and Symptom Distress Scale-59R were also recorded at 12 months, 24 months, and 36 months posttransplant in the BENEFIT study to determine patient self-reported side effects. A direct relationship was seen between patients receiving a transplant and an increase in their overall HRQoL. The patients in the BENEFIT study had an increase in their physical and mental composite score, which reached scores similar to the general population at 12 months posttransplant and continued until at least 36 months when the study ended. Similar results were depicted in the BENEFIT-EXT group but to a lesser degree. The BENEFIT-EXT group obtained scores comparable to the general population scores only for the physical composite scores. The study showed that worsening kidney function led to clinically significant decline in QoL, excluding BENEFIT at 12 months and BENEFIT-EXT at 36 months. An increase 
in the number of side effects correlated with a clinically significant reduction in the patients' physical and mental composite scores at 36 months. Statistically and clinically significant differences were seen in absolute physical composite scores for patients treated with belatacept in both the BENEFIT and BENEFIT-EXT studies, 1.7-2.1 points and 2.3-2.8 points, respectively; however, there was no difference in the mental composite scores. The study ultimately determined that HRQoL improved after kidney transplantation to match that of the general population, and patients who used belatacept as their maintenance immunosuppressive therapy reported fewer side effects, resulting in improved QoL, compared to patients on CsA.

\section{Renal outcomes}

Funding and time are two luxuries that are of limited supply in the research realm; therefore, the research community continues to develop surrogate markers that can better predict a clinical outcome without requiring multiple, longterm studies to be conducted. Schnitzler et $\mathrm{a}^{25}$ conducted a retrospective analysis to determine whether the eGFR value 1 year after transplant can accurately predict future loss of a patient's allograft. In the study, 87,575 patients' medical records were accessed, which included 345 patients from the BENEFIT and 244 patients from the BENEFIT-EXT studies. The study models used the structure and covariates developed by the United Network for Organ Sharing Kidney Allocation Committee to predict survival after kidney transplantation. ${ }^{26}$ The eGFR, calculated according to the Modification of Diet in Renal Disease equation, at 12 months posttransplant proved to be a statistically significant predictor of all-cause graft mortality for all types of kidney donors $(P<0.001)$ by using multivariate Cox regression analyses for relative hazards of graft loss at 12 months compared to baseline. The authors predicted graft survival of patients in the BENEFIT and BENEFIT-EXT studies for years 2-9 as a $3.9 \%$ and $4.2 \%$ mean difference in the LI belatacept arm compared to the CsA arm, respectively. Observed differences in the studies showed $4.2 \%$ differences in graft loss in BENEFIT and 1.4\% in BENEFIT-EXT. The model was directed toward all-cause graft survival as well, where observations were noted to be similar. Predicted graft survival was $90.7 \%$ for LI belatacept and $86.5 \%$ for CsA group for the BENEFIT study, while the observed values were $92 \%$ and $88.7 \%$ for the LI belatacept and CsA at 3 years, respectively. Results were similar for BENEFIT-EXT, with slightly lower survival rates. This study validated that there is a relationship between renal function and graft loss as well as patient survival; therefore, eGFR is an appropriate surrogate marker to predict clinically significant outcomes in relation to kidney transplant recipients.

\section{Cardiovascular risk}

A study conducted by Soveri et $\mathrm{al}^{27}$ evaluated the external validity of the cardiovascular risk equation and its application to the BENEFIT and BENEFIT-EXT studies. This cited study recognized that the Framingham risk score is unable to adequately predict the risk of cardiovascular disease in kidney transplant recipients. Instead, the 7-year major adverse cardiac event (MACE) was used. This risk calculator was developed by a multicenter clinical trial (ALERT) and previously validated by data in the Patient Outcomes in Renal Transplantation (PORT) observational study. Incidence of diabetes mellitus, low-density-lipoprotein cholesterol, and serum creatinine at 3 years in the BENEFIT and BENEFITEXT studies was used in these models. Using these risk calculator estimations, belatacept may result in a substantial reduction in MACE $(>20 \%)$ and mortality $(\sim 18 \%-30 \%)$. Long-term follow-up is needed to determine whether these estimates will predict mortality.

\section{Pharmacoeconomics}

A $250 \mathrm{mg}$ vial of the medication is estimated to cost $\$ 1,107$, based on the average wholesale price. Two to three vials are needed for a typical patient's dosage. Alternative immunosuppressive cost would include CNIs and CNI monitoring. Published analyses are needed to determine how the belatacept drug cost and infusion costs compare to the potential improvements in safety and efficacy.

\section{Conclusion}

Overall, allograft survival is comparable for patients receiving either belatacept or CsA as maintenance immunosuppression. In contrast, early acute rejection rates were significantly higher in the MI belatacept treatment arm and numerically higher in the LI arm of the BENEFIT study when compared to CsA. In the BENEFIT-EXT study, rejection rates were similar in the belatacept and CsA treatment arms. Overall, belatacept resulted in a higher average eGFR compared to the CsA regimen, an outcome that was sustained throughout longterm follow-up studies. In the BENEFIT study, SCDs showed immediate improvement in renal function with FDA-approved doses of belatacept (LI) when compared to administration of CsA. In the BENEFIT-EXT study, significant improvement in ECDs was delayed until 5 years after transplantation with the FDA-approved dosing (LI) when compared to CsA dosing. 
Summative evidence of the trials reveals lower levels of blood pressure, cholesterol, and incidence of NODAT with belatacept compared to CsA-based regimens. Cases of PTLD, PML, and malignancies were reported, but similar incidences occurred with the various treatment regimens; therefore, it cannot be confirmed whether belatacept was the causative agent. The development of donor-specific antibodies was lower with belatacept-based regimens.

Belatacept should not be used in patients who are EBV seronegative. Further studies are needed to determine which patients will benefit the most from belatacept-based regimens. Patients at risk for diabetes, hypertension, and dyslipidemia may benefit from the minimal side effect profile of belatacept. Long-term data are needed to determine whether these benefits will translate into improved survival/decreased mortality. Patients must be willing to receive, tolerate, and afford monthly infusions. In summary, belatacept is a safe and effective alternative to first-line CNIs, which maintains similar allograft survival in kidney transplant recipients while improving renal function. Additional studies are required to validate the efficacy of belatacept compared to tacrolimus, in addition to studies that formally analyze the relationship between belatacept therapy and the development of PTLD and PML. Conversion therapy and surrogate markers of definitive end points need to be further studied.

\section{Disclosure}

The authors report no conflicts of interest in this work.

\section{References}

1. Kidney Disease: Improving Global Outcomes (KDIGO) Transplant Work Group. KDIGO clinical practice guideline for the care of kidney transplant recipients. Am J Transplant. 2009;9(Suppl 3):S1-S155.

2. Belatacept [Package Insert] [webpage on the Internet]. Highlights of Prescribing Information. 2016. Available from: http://packageinserts. bms.com/pi/pi_nulojix.pdf. Accessed March 8, 2016.

3. Sam T, Gabardi S, Tichy EM. Risk evaluation and mitigation strategies: a focus on belatacept. Prog Transplant. 2013;23(1):64-70.

4. Grinyó J, Charpentier B, Pestana JM, et al. An integrated safety profile analysis of belatacept in kidney transplant recipients. Transplantation. 2010;90(12):1521-1527.

5. Vincenti F, Larsen C, Durrbach A, et al. Costimulation blockade with belatacept in renal transplantation. NEJM. 2005;353:770-781.

6. Vincenti F, Charpentier B, Vanrenterghem Y, et al. A phase III study of belatacept-based immunosuppression regimens versus cyclosporine in renal transplant recipients (BENEFIT study). Am J Transplant. 2010;10(3):535-546.

7. Durrbach A, Pestana JM, Pearson T, et al. A phase III study of belatacept versus cyclosporine in kidney transplants from extended criteria donors (BENEFIT-EXT study). Am J Transplant. 2010;10(3):547-557.

8. Nulojix. [webpage on the Internet]. Risk evaluation and mitigation strategy for increased risk of PTLD, predominantly involving the CNS, and PML with NULOJIX. Available from http:/www.nulojix.com/ hcp/rems.aspx?cm_mc_uid=03785373987414557419388\&cm_mc_ sid_51110000=1459792841. Accessed 4 April 2016.
9. Vincenti F, Blancho G, Durrbach A, et al. Five-year safety and efficacy of belatacept in renal transplantation. J Am Soc Nephrol. 2010;21(9): 1587-1596.

10. Larsen CP, Grinyó J, Medina-Pestana J, et al. Belatacept-based regimens versus a cyclosporine A-based regimen in kidney transplant recipients: 2-year results from the BENEFIT and BENEFIT-EXT studies. Transplantation. 2010;90(12):1528-1535.

11. Vincenti F, Larsen CP, Alberu J, et al. Three-year outcomes from BENEFIT, a randomized, active-controlled, parallel-group study in adult kidney transplant recipients. Am J Transplant. 2012;12: 210-217.

12. Rostaing L, Vincenti F, Grinyó J, et al. Long-term belatacept exposure maintains efficacy and safety at 5 years: results from the long-term extension of the BENEFIT study. Am J Transplant. 2013; 13(11):2875-2883.

13. Vincenti F, Rostaing L, Grinyo J. Belatacept and long-term outcomes in kidney transplantation. N Engl J Med. 2016;374(4):333-343.

14. Pestana JO, Grinyo JM, Vanrenterghem Y, et al. Three-year outcomes from BENEFIT-EXT: a phase III study of belatacept versus cyclosporine in recipients of extended criteria donor kidneys. Am J Transplant. 2012;12(3):630-639.

15. Charpentier B, Pestana JO, del C, et al. Long-term exposure to belatacept in recipients of extended criteria donor kidneys. Am J Transplant. 2013;13(11):2884-2891.

16. Florman S, Pestana J, Rial M, et al. Final results from the BENEFIT-EXT trial: a 7-year follow-up of belatacept treated patients. Am JTransplant. 2015;15(S3):1. abstract 455 .

17. Rostaing L, Neumayer HH, Reyes-Acevedo R, et al. Belataceptversus cyclosporine-based immunosuppression in renal transplant recipients with pre-existing diabetes. Clin J Am Soc Nephrol. 2011;6(11):2696-2704.

18. Ferguson R, Grinyó J, Vincenti F, et al. Immunosuppression with belatacept-based, corticosteroid-avoiding regimens in de novo kidney transplant recipients. Am J Transplant. 2011;11(1):66-76.

19. Masson P, Henderson L, Chapman JR, Craig JC, Webster AC. Belatacept for kidney transplant recipients. Cochrane Database Syst Rev. 2014;11:CD010699.

20. Kirk AD, Guasch A, Xu H, et al. Renal transplantation using belatacept without maintenance steroids or calcineurin inhibitors. Am J Transplant. 2014;14(5):1142-1151.

21. Rostaing L, Massari P, Garcia VD, et al. Switching from calcineurin inhibitor-based regimens to a belatacept-based regimen in renal transplant recipients: a randomized phase II study. Clin J Am Soc Nephrol. 2011;6(2):430-439.

22. Grinyo J, Alberu J, Contieri FL, et al. Improvement in renal function in kidney transplant recipients switched from cyclosporine or tacrolimus to belatacept: 2-year results from the long-term extension of a phase II study. Transpl Int. 2012;25(10):1059-1064.

23. Gupta G, Regmi A, Kumar D, et al. Safe conversion from tacrolimus to belatacept in high immunologic risk kidney transplant recipients with allograft dysfunction. Am J Transplant. 2015;15(10): 2726-2731.

24. Dobbels F, Wong S, Min Y, Sam J, Kalsekar A. Beneficial effect of belatacept on health-related quality of life and perceived side effects results from the BENEFIT and BENEFIT-EXT trials. Transplantation. 2014;98(9):960-968.

25. Schnitzler MA, Lentine KL, Axelrod D, et al. Use of 12-month renal function and baseline clinical factors to predict long-term graft survival application to BENEFIT and BENEFIT-EXT trials. Transplantation 2012;93(2):172-181.

26. Wolfe RA, McCullough KP, Schaubel DE, et al. Calculating life years from transplant (LYFT): methods for kidney and kidney pancreas candidates. Am J Transplant. 2008;8(4Pt 2):997-1011.

27. Soveri I, Snyder J, Holdaas H, et al. The external validation of the cardiovascular risk equation for renal transplant recipients: applications to BENEFIT and BENEFIT-EXT trials. Transplantation. 2013;95(1): $142-147$. 


\section{Publish your work in this journal}

The International Journal of Nephrology and Renovascular Disease is an international, peer-reviewed open-access journal focusing on the pathophysiology of the kidney and vascular supply. Epidemiology, screening, diagnosis, and treatment interventions are covered as well as basic science, biochemical and immunological studies. The journal welcomes

original research, clinical studies, reviews \& evaluations, expert opinion and commentary, case reports and extended reports. The manuscript management system is completely online and includes a very quick and fair peerreview system, which is all easy to use. Visit http://www.dovepress.com/ testimonials.php to read real quotes from published authors.

Submit your manuscript here: http://www.dovepress.com/international-journal-of-nephrology-and-renovascular-disease-journal 